\title{
Patient-Reported Outcomes of Retropubic versus Trans-Obturator Mid-Urethral Slings for Urinary Stress Incontinence: The Malaysian Experience
}

\author{
Zalina $\mathrm{N}^{\mathrm{a}}$, Kamal $\mathrm{N}^{\mathrm{b}}$, Aruku $\mathrm{N}^{\mathrm{b}}$, Jamaluddin $\mathrm{AR}^{\mathrm{c}}$ \\ a Obstetrics \& Gynaecology Department, Kulliyyah of Medicine, International Islamic University Malaysia \\ ${ }^{b}$ Obstetrics \& Gynaecology Department, Hospital Ipoh, Perak, Malaysia \\ c Department of Community Health, Kulliyyah of Medicine, International Islamic University Malaysia
}

\begin{abstract}
Introduction: Studies reported that trans-obturator mid-urethral sling (TOT) is superior in safety and efficacy when compared with retro-pubic trans-vaginal tape (TVT) with regard to bladder, vascular and urethral injury. The purpose of this study is to determine if the TOT is equivalent to the TVT by using patient-reported outcomes for the treatment of urodynamic stress urinary incontinence (USI) at Ipoh Hospital. Methods: It is a cross-sectional study on all consecutive patients who underwent TVT procedure between Jan 2005 and Jan 2006 and TOT procedure from Jan 2006 to Jun 2008. Post operatively; all women were followed up at one month, three months, 12 months and annually, thereafter. Data was retrieved from the patients' records based on the standard pre-operative assessment sheet and follow up record. Then, interviews were made via a telecommunication to assess the patients' symptoms and satisfaction using a validated questionnaire. Data analysis were performed using IBM SPSS Statistics for Windows version 19 (SPSS Inc., Chicago, Illinois) considering a significant level of $P<0.05$. Results: Total of 51 subjects in TVT group and 52 in TOT group were available for analysis. At 12 months, the stress urinary incontinence (SUI) cure rate is higher in the TVT (90.2\%) compared to TOT $(80.8 \%)$ group; however, it was not statistically significant $(\mathrm{p}=0.279)$. The lower urinary tract symptoms (LUT) were improved post-operatively in both groups with urgency and urgency incontinence being significantly better in the TVT group. Four $(7.4 \%)$ subjects in the TVT group had a bladder perforation $(p=0.992)$ and one case of vaginal erosion reported in the TOT group. Conclusion: TVT had a higher successful rate in the treatment of SUI; however, it increases the risk of bladder perforation. Over active bladder symptom (OAB) were significantly improved in the TVT compared to TOT group.
\end{abstract}

KEYWORDS: Trans-obturator mid-urethral slings, retro-pubic mid-urethral slings, stress urinary incontinence, overactive urinary bladder.

\section{INTRODUCTION}

Urinary incontinence is common in women with reported prevalence varying from $10-46 \%$ in different populations. ${ }^{1}$ It is thought to result from a loss of pubo-urethral ligamentous support to the urethra. The main aim of modern incontinence surgery is to correct this by recreating the mid-urethral support. ${ }^{2}$

TVT was the first mid-urethral sling (MUS) introduced by Ulmsten and Petros in 1995 with a cure rate ranging from 84 to $95 \% .{ }^{3,4,5}$ Although highly successful, TVT procedure can be complicated by injuries to the

Corresponding author:

Zalina Nusee,

M.D (UKM), M.O.G (UKM), Fellow Urogynaecology

(Taiwan)

Department of Obstetrics \& Gynaecology,

International Islamic University Malaysia,

Kuantan Campus, Jalan Hospital,

25312 Kuantan, Pahang, Malaysia.

Email:drzalina@iium.edu.my intra-peritoneal structures due to the blind passage of trocars through the retro-pubic space. The most common complication, bladder perforation, is reported to occur in 2.7 to $30 \%$ cases. 5,6 Serious injuries such as bowel perforations, and neurovascular injuries is rare, but has been reported. ${ }^{7,8}$

In 2001, a new surgical technique of 'OAB' (MUS) prosthetic suspension called the trans obturator tape was introduced by Delorme in an attempt to reduce the intraoperative complications. ${ }^{9}$ In contrast to the retro-pubic approach, the transobturator approach involves the passage of a sub-urethral sling through the medial aspect of the obturator membrane. The advantage is that there being a greater distance to vital structures such as the bladder and intra-peritoneal contents such as the bowel. In addition, due to the comparatively less acute angle of the sling, it was suggested that trans obturator sling could potentially be less obstructive to urine flow through the urethra. Although systematic reviews have confirmed the efficacy of MUS, 5-20\% of treated patients do experience surgical failure with persistent or recurrent USI. ${ }^{6}$ 
To date, in our local setup, the comparative study between TVT and TOT is scarce since it was first introduced here in 2002 and 2005 respectively. The purpose of this study is to determine if the TOT is equivalent to the TVT by using patient-reported outcomes for the treatment of USI in Ipoh Hospital.

\section{MATERIALS AND METHODS}

This is a cross-sectional study on all women who underwent a TOT procedure between January 2006 and June 2008, or TVT procedure between Jan 2005 and Jan 2006 in Ipoh Hospital. After obtaining the institutional review board approval, a surgical database for the patients operated on the dates above by the third author was accessed. Surgery was only offered if conservative therapy was unsuccessful in all urodynamic proven SUI. Women with concomitant problems (e.g. utero-vaginal prolapse, anterior and posterior vaginal wall prolapsed) were also included into the study except those with a predominant urgency incontinence (UUI) and urodynamic bladder over activity (DO). All patients in the study group underwent a history taking and pelvic examination. The preoperative investigations include urine dipstick and cultures, urodynamic examination (cystometry and uroflometry), and post-void residual volume (PVR) measurement. The TOT (Monarc, American Medical Systems (AMS)) and TVT (Johnson \& Johnson (Gynaecare)) procedures were performed by the third author according to the technique of Delorme and Ulmsten using Uratape (Mentor-Porgès). ${ }^{4,9}$

Post-operatively, they were followed up at one month, three months, 12 months and annually, thereafter. Post-operative cure is defined by disappearance in SUI symptoms and objectively, when there was negative stress cough. Cough stress test was performed during each follow up with a minimum bladder volume of $200 \mathrm{ml}$. Subjective cure was considered in those women who responded "no" to the question of leaking urine during physical activity, coughing, or sneezing (appendix 1, question 1 in the questionnaire). The exclusion criteria include; incomplete data, loss of patient's record, defaulters and those who were non-contactable.

Data analysis was performed using IBM SPSS Statistics for Windows version 19 (SPSS Inc., Chicago, Illinois). Normally distributed numerical data were presented in mean and standard deviation. Categorical data were described using count and percentage. Independent sample t-test was used to compare two means while chi-square or Fisher's exact test were used to compare two qualitative variables. Significance was taken at $P<0.05$.

Flow chart of the study design

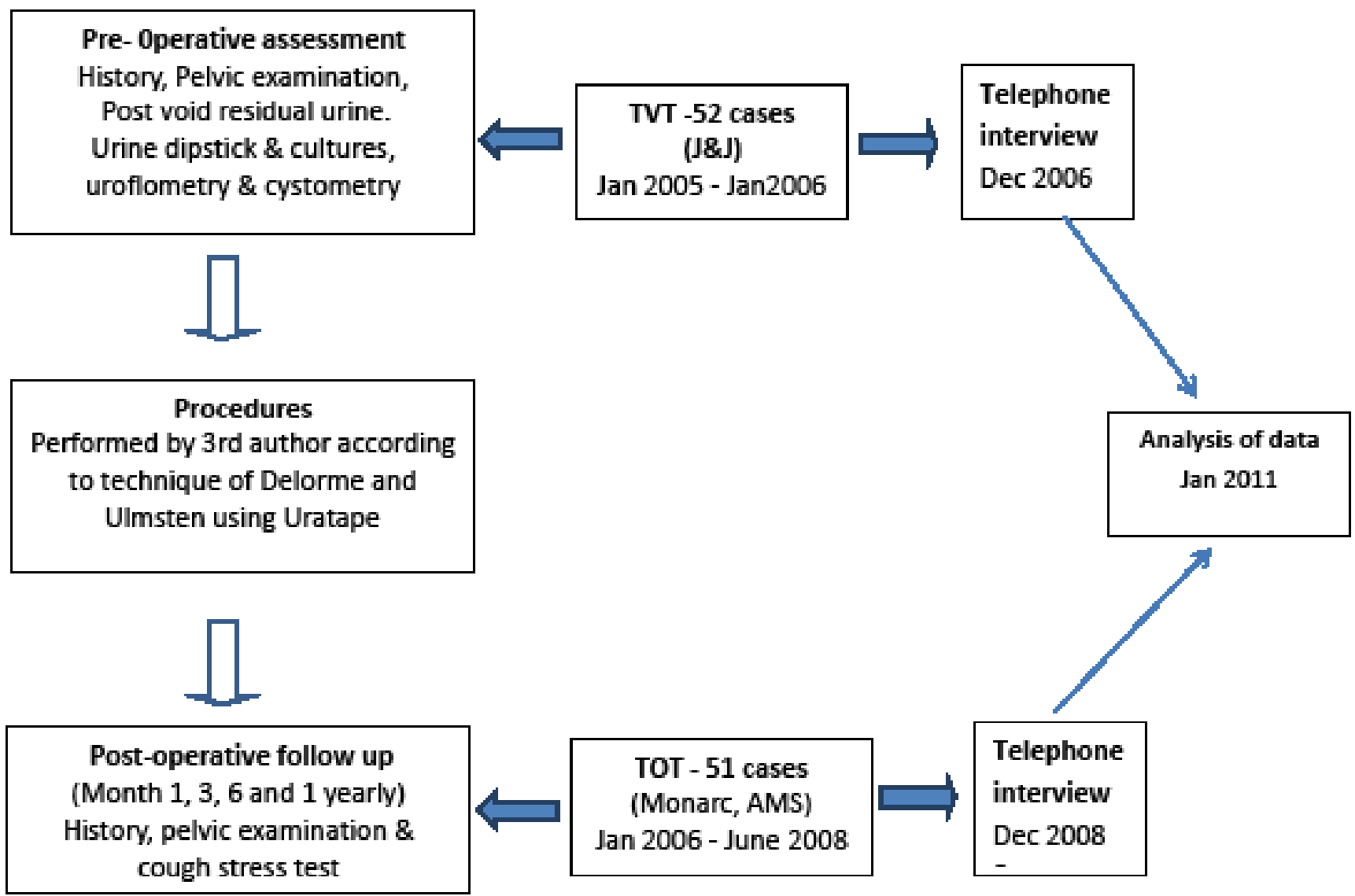


There were a total of 51 and 52 available data for analysis from the TOT and TVT procedures, respectively. Patients' demographic data were similar in both groups as shown in Table I. The mean time interval between the operation and telephone interview was 16 months for TOT and 12 months for TVT groups. TVT group reported more symptoms of UUI preoperatively, which was $78.4 \%(p=0.118)$.

Table I. Patient characteristics

\begin{tabular}{|c|c|c|c|}
\hline Characteristic & TVT $(n=51)$ & TOT $(n=52)$ & $P$ \\
\hline Age (mean) & $56.98(\mathrm{SD}=10.39)$ & $54.3(\mathrm{SD}=10.60)$ & 0.901 \\
\hline \multicolumn{4}{|l|}{ BMI } \\
\hline Normal (18.5-22) & $9(17.6 \%)$ & $12(22.2 \%)$ & 0.441 \\
\hline Over weight (23-27.5) & 27 (52.9\%) & $21(40.7 \%)$ & \\
\hline Obese $(>27)$ & $15(29.5 \%)$ & $19(33.3 \%)$ & \\
\hline \multicolumn{4}{|l|}{ Ethnic } \\
\hline Malay & $19(37.3 \%)$ & $22(42.3 \%)$ & 0.849 \\
\hline Chinese & $14(27.4 \%)$ & $14(26.9 \%)$ & \\
\hline Indian & $18(35.3 \%)$ & $16(30.8 \%)$ & \\
\hline \multicolumn{4}{|l|}{ Parity } \\
\hline Nullipara & 0 & $5(9.6 \%)$ & 0.070 \\
\hline Multiparity & $51(100 \%)$ & $47(90.4 \%)$ & \\
\hline \multicolumn{4}{|l|}{ Menopausal status } \\
\hline Premenopausal & $18(35.3 \%)$ & $21(40.4 \%)$ & 0.742 \\
\hline Postmenopausal & $33(64.7 \%)$ & $31(59.6 \%)$ & \\
\hline Previous hysterectomy & $6(11.7 \%)$ & $13(25.0 \%)$ & 0.139 \\
\hline Previous prolapse surgery & $8(15.6 \%)$ & $5(9.6 \%)$ & 0.528 \\
\hline Previous incontinence surgery & $4(9.8 \%)$ & 0 & 0.063 \\
\hline \multicolumn{4}{|l|}{ Diagnosis } \\
\hline Incontinence only & $26(51.0 \%)$ & 27 (51.9\%) & 0.924 \\
\hline Incontinence \& POP & $25(49.0 \%)$ & $25(48.1 \%)$ & \\
\hline \multicolumn{4}{|l|}{ Procedure } \\
\hline SUS without anterior repair & $34(66.7 \%)$ & $32(61.5 \%)$ & 0.588 \\
\hline SUS with VTH and A-P repair & $17(33.3 \%)$ & $20(38.5 \%)$ & \\
\hline
\end{tabular}

POP: pelvic organ prolapsed, VTH: vaginal total hysterectomy, A-P repair: anterior and posterior vaginal repair, SUS: sub urethral sling

The objective SUI cure rate for the TVT and TOT were $90.2 \%$ and $80.8 \%(p=0.279)$ respectively, based on the cough stress test (Table II). From the patients' telephone interviews using specific questionnaires, e.g. referring to question number 1 (appendix 1): "Do you have urinary leakage when you cough, sneeze, conduct physical activity? Do you have the symptoms now?" $86.5 \%(45 / 52)$ and $96.0 \%$ (49/51) women from TOT and TVT answered 'no' respectively. Forty nine women $(94.2 \%)$ from TOT were satisfied with the procedure compared to 50 women $(98.0 \%)$ in the TVT group. 
Table 2. Lower Urinary Tract Symptoms

\begin{tabular}{llllll}
\hline \multirow{2}{*}{ Symptoms } & \multicolumn{2}{c}{ TVT $(\mathrm{N}=51)$} & \multicolumn{2}{c}{ TOT $(\mathrm{N}=52)$} & \\
\cline { 2 - 4 } & $\begin{array}{l}\text { Pre-Op } \\
\mathrm{n}(\%)\end{array}$ & $\begin{array}{l}\text { Post-Op } \\
\mathrm{n}(\%)\end{array}$ & $\begin{array}{l}\text { Pre-Op } \\
\mathrm{n}(\%)\end{array}$ & $\begin{array}{l}\text { Post-Op } \\
\mathrm{n}(\%)\end{array}$ & $P^{*}$ \\
\hline SUI & $51(100)$ & $5(9.80)$ & $52(100)$ & $10(19.20)$ & 0.279 \\
Urge incontinence & $40(78.4)$ & $1(1.96)$ & $16(30.76)$ & $10(25.00)$ & $<0.001$ \\
Frequency & $48(94.1)$ & $11(21.57)$ & $24(46.15)$ & $7(13.46)$ & 0.782 \\
Urgency & $36(70.6)$ & $2(3.92)$ & $24(46.15)$ & $11(21.15)$ & 0.005 \\
Voiding difficulty & $9(17.7)$ & 0 & $13(25.00)$ & $3(5.76)$ & 0.280 \\
Incomplete empty & $30(58.8)$ & 0 & $16(30.76)$ & $3(5.76)$ & 0.053 \\
\hline
\end{tabular}

* Fisher's exact test

The LUT symptoms, identified as frequent urgency, voiding difficulty, and incomplete voiding were significantly improved following both procedures. Post-operatively, TVT group showed significant improvement in UUI $(97.5 \%$ vs. $37.5 \%)$ and urgency (94.4\% vs. $54.2 \%)$ compared to TOT (Table 2$)$. Three women $\mathbf{( 5 . 7 \% )}$ developed a new urgency symptom (de novo) following TOT. There were no reported cases of bladder perforation following TOT as shown in Table III. However, all the complications were not statistically different between the two groups.

Table 3. Complications of MUS procedure

\begin{tabular}{llll}
\hline & $\begin{array}{l}\text { TOT }(\%) \\
\mathrm{N}=52\end{array}$ & $\begin{array}{l}\text { TVT }(\%) \\
\mathrm{N}=51\end{array}$ & $P^{*}$ \\
\hline Bladder perforation & 0 & $4(7.8)$ & 0.150 \\
Urinary retention & $2(3.8)$ & $7(13.7)$ & 0.133 \\
Infection/UTI & $1(1.9)$ & $4(7.8)$ & 0.088 \\
Mesh erosion & $1(1.9)$ & 0 & 0.992 \\
\hline
\end{tabular}

* Fisher's exact test

\section{DISCUSSION}

In a large, multi-center RCT using a composite of objective and subjective outcomes, the success rate for TVT procedures was higher, $80.8 \%$ compared to $77 \%$ for TOT. ${ }^{10}$ This is consistent with our findings, which revealed TVT was superior in terms of cure rate both subjective (96.0\% vs.86.5\%) and objectively (90.2\% vs. $80.8 \%)$. The contributing factor could be related to the hammock, and $U$ shape position of the sling.
In a recent multi-center randomized study, patientreported outcomes have shown that TOT is not inferior to TVT, but both produce lower success rates than studies using objective measures. ${ }^{11}$ We found that the subjective cure rate is higher in both groups. This could be due to the time difference between the two in which the subjective assessment was performed later. The cure rate is higher after the presence of 
tissue in growth in between the mesh which enhances the back board effect in supporting the urethra.

There is only one randomized controlled trial which has shown an improvement in OAB symptoms, i.e. a $96 \%$ cure rate for urgency symptoms in both groups. ${ }^{12}$ Only 2.3\% (three patients in each group) developed new onset urgency post-operatively. All other studies (mainly retrospective cohorts) have shown no differences between TVT and TOT for concomitant urgency or UUI.

In our study, despite having more UUI and frequency symptoms preoperatively, TVT was reported as a significant improvement of these symptoms compared to the TOT group. This contradicts the theory of increased OAB or de novo urgency in TVT group in view of its close approximates with the bladder. Subjective assessment revealed that three women in the TOT group complained of a new onset of urgency.

Richter et al. reported higher rates of voiding difficulty with retropubic TVT compared with TOT $(2.7 \%$ vs. $0 \%) .{ }^{10}$ It is further supported by Porena et al. who found voiding difficulty in $44 \%$ and $24 \%$ following TVT and TOT, respectively. ${ }^{13}$ In this study, we observed that TVT has four times higher chance of urinary retention compared to TOT $(13.7 \%$ vs. $3.8 \%)$. This is possibly because it is more obstructive nature of the TVT procedure. However, definitions of voiding difficulty vary between studies, thus, making it difficult to draw conclusions or make recommendations.

One study found a higher rate of bladder perforation in the TVT group compared with the TOT. ${ }^{14}$ This is consistent with this study; four cases of bladder perforation occurred in TVT and none following TOT. This could be related to the learning curve of the surgeon. TVT procedure was the first MUS procedure performed at the beginning of his urogynecological carrier followed by TOT two years later. Although the incidence of bladder puncture did not reach a statistical significance level, the potential for serious sequelae does need to be considered. There were no serious resultant complications observed in our cases. Mesh protrusion rates were higher in the TOT group, which may have been as a result of inadvertent, unrecognized vaginal wall puncture.

There were some limitations in this study in which it was not randomized and performed separately at two different times. The severity and type of SUI were not accessed, which could affect the outcome between two groups. We have limited number of women who have isolated SUI in our center. Therefore, we have to include cases with other concomitant surgeries, which might not reflect the real outcome of MUS procedures. TVT procedure was carried out at the beginning of the 3rd author's Urogynaecology work that could have been contributed to the higher morbidity in TVT group compared to TOT.

\section{CONCLUSION}

The TVT has higher objective and subjective cure rate of SUI but also with higher risk of bladder perforation and urinary obstruction. The subjective cure rate is higher than objective cure rate in both groups. Both TVT and TOT significantly improved LUT symptoms with OAB being more significantly different in TVT.

\section{REFERENCES}

1. Hannestad YS, Lie RT, Rortveit G, Hunskaar S. Familial risk of urinary incontinence in women: population based cross-sectional study. BMJ 2004; 329: 889-91.

2. Nilsson CG, Kuuva N. The tension-free vaginal tape procedure is successful in the majority of women with indications for surgical treatment of urinary stress incontinence. BJOG 2001; 108:414-9.

3. Ulmsten U, Petros P. Intravaginal slingplasty (IVS): an ambulatory surgical procedure for treatment of female urinary incontinence. Scand J Urol Nephrol 1995; 29:75-82.

4. Ulmsten U, Johnson P, Rezapour M. A three-year follow up of tension free vaginal tape for surgical treatment of female stress urinary incontinence. Br J Obstet Gynaecol1999; 106:345-50.

5. Debodinance P, Delporte P, Engrand JB, et al. Tension-free vaginal tape (TVT) in the treatment of urinary stress incontinence: 3 years experience involving 256 operations. Eur J Obstet Gynecol Reprod Biol 2002; 105:49-58.

6. Meschia M, Pifarotti P, Bernasconi F, et al. Tension-Free vaginal tape: analysis of outcomes and complications in 404 stress incontinent women. Int Urogynecol J Pelvic Floor Dysfunct 2009; 12: S24-S27.

7. Novara G, Ficcara V, Boscolo-Berto R, et al. Tension-free midurethral slings in the treatment of female stress urinary incontinence: a systematic review and meta-analysis of randomized controlled trial of effectiveness. Eur Urol 2007; 52:663-79.

8. Koops SE, Bisseling TM, Van Brummen HJ, Heintz $A P$, Vervest HA. What determines a successful tension-free vaginal tape? A prospective multicenter cohort study: results from The Netherlands TVT database. Am J Obstet Gynecol 2006; 194: 65-74.

9. Delorme E. Transobturator urethral suspension: mini invasive procedure in the treatment of stress urinary incontinence in women. Prog Urol 2001; 11:1306-13. [French]

10. Richter HE, Albo ME, Zyczynski HMD, et al. Retropubic versus transobturator midurethral slings for stress incontinence. N Engl J Med 2010; 362:2066-76. 
11. Freeman R, David Holmes D, Hillard T, et al. What patients think: patient-reported outcomes of retropubic versus trans-obturator mid-urethral slings for urodynamic stress incontinence a multi-centre randomised controlled trial. Int Urogynecol J 2011; 22:279-86.

12. Laurikainen E, Valpas A, Kivelä A, et al. Retropubic compared with transobturator tape placement in treatment of urinary incontinence: a randomized controlled trial. Obstet Gynecol 2007; 109:4-11.

13. Porena M, Costantini E, Frea B, et al. Tension-free vaginal tape versus transobturator tape as surgery for stress urinary incontinence: results of a multicentre randomised trial. Eur Urol 2007; 52:1481-90.

14. Barry C, Lim YN, Muller R, et al. A multi-centre, randomised clinical control trial comparing the retropubic (RP) approach versus the transobturator approach (TO) for tension-free, suburethral sling treatment of urodynamic stress incontinence: the TORP study. Int Urogynecol J Pelvic Floor Dysfunct 2008; 19:171-8.

\section{Appendix 1}

\section{MUS Operation Follow Up Questionnaire}

Name:

Date of Birth:

\section{Time Since MUS Operation:}

Do you have:

1) Urinary stress incontinence (urine leakage when you cough, sneeze, conduct physical activity) Present before the operation: yes / no Do you have this symptom now: yes / no

2) Urinary frequency (having to empty your bladder very often)

Present before the operation: yes / no Do you have this symptom now: yes / no

3) Urinary urgency (sudden onset strong, compelling desire to empty your bladder)

Present before the operation: yes / no

Do you have this symptom now: yes / no

4) Urinary leakage as a result of urinary urgency (i.e. not getting to toilet in time)

Present before the operation: yes / no

Do you have this symptom now: yes / no

5) Difficulty emptying your bladder (eg. having to strain to pass urine, difficulty getting started, poor urine stream) Present before the operation: yes / no Do you have this symptom now: yes / no
6) The bladder does not feel empty after passing urine (eg. When you stand up you start dribbling some urine, need to void again within 5-10 minutes)

Present before the operation: yes / no Do you have this symptom now: yes / no

7) Pain or discomfort in the lower abdominal or groin area

Present before the operation: yes / no Do you have this symptom now: yes / no

8) Over the last week, how would you rate your continence?

(a) Completely dry

(b) Substantially continent: may have periodic leakage of small amounts, but additional protection is not needed

(c) Some protection may be required: needing up to 3 pads per day, or when leakage occur not more than once weekly

(d) Substantially or totally incontinent: requiring more than 3 pads a day, or large leak 2 or more times a week

9) Are you satisfied with the result of the operation? Yes / no

10) Any additional comments? 